\title{
A Comparative of SIFT and SURF Features for Stitching Aerial Images
}

\author{
Raden Sumiharto ${ }^{1}$, Agus Harjoko ${ }^{2}$ and Agfianto Eko Putra ${ }^{3}$ \\ ${ }^{1,2,3}$ Departement of Computer Science and Electronics, Universitas Gadjah Mada, \\ Yogyakarta, Indonesia \\ ${ }^{1}$ __sumiharto@ugm.ac.id, ${ }^{2}$ aharjoko@ugm.ac.id, ${ }^{3}$ agfi@ugm.ac.id
}

\begin{abstract}
SIFT and SURF as feature detection methods are already widely used in various applications. Stitching of aerial images can be based on image features. This paper discusses the use of SIFT and SURF features for stitching aerial images. A number of parameters affecting the stitching algorithm are observed, such as the height of image acquisition (scale between image) and area of overlap between image. Aerial images acquired at difference altitude resulting in a different scaling and thus object resolution. Altitude difference and angle difference (due to flight control) resulting in a different overlapping area of the images to be stitched. So that both methods can be used for stitching process image aerial. The results comparison of the two methods shows that the difference in height and the area of overlap slightly (4\%) and scale difference between images by $45 \%$ to $480 \%$ by SIFT and $25 \%$ to $600 \%$ by SURF is still possible to process image aerial stitching.
\end{abstract}

Keywords: Overlap, scaling, image feature

\section{Introduction}

Aerial photography is captured an area of a certain height, but still within the atmospheric space. Results of images taken by the camera of a fixed wing are usually not enough to cover the area that serves as the object of aerial photography for mapping purposes because the process aerial photography done gradually because it does not allow the aircraft to stop somewhere just to find the right position to make decision aerial photographs. So that the resulting image only has a certain sweep and fragmented between photos with each other. To get broader picture results can be done so that the image merging technique to an image that is larger and covers all areas that become the object of aerial photographs. Steps that can be used in combining aerial photographs are using SIFT (Scale-invariant Feature Transform) and SURF (Speeded-up Robust Features) method. Both of these methods can produce an output image with a broader view that consisted of a combination of several images. Both methods can be compared to variations in translation, rotation and scaling on the stitching process. SIFT [1,2,3] (Scale Invariant Feature Transform) is a feature point detection and matching algorithm proposed by Lowe in 1999. The SIFT features are not only invariant to image scaling, translation, and rotation but also partially invariant to illumination changes and affine transformation. The SIFT algorithm which is based on feature recognition and matching is used in many fields, such as object recognition, image mosaic, mobile robot localization and map building. Lowe used the method of comparing the distance of the closest neighbor to that of the second-closest neighbor to match the key point. When the ratio is less than the threshold, it can be considered to be the correct matching. When reducing the threshold, matching points will decrease, but little mismatches. When raising the

Received (May 25, 2017), Review Result (August 28, 2017), Accepted (September 1, 2017) 
threshold, matching points will increase, but there will be a lot of mismatches. Usually, we want feature points matched correctly the more the better. In this case, we need to increase the matching threshold, but following there will be a lot of mismatches. In order to solve this problem, actually, we need to find a robust estimation method, which should estimate the parameters to build the model to classify the data containing many outlier data. The researchers recommended some strong robust estimation method [4,5]; one of the most representatives is the RANSAC [6] algorithm. But when the data space contains a lot of mismatches, finding the right transformation matrix will be very difficult, and the computer needs lots of time to calculate the parameters.

Speeded-up Robust Features of SURF [7,8,9,10]is a robust image detection, first in presented by Herbert Bay in 2006, is widely used in computer vision as an object identifier, or a 3D reconstruction algorithm SURF produce high-dimensional image features. SURF is point features of an image. Point features are points of an image whose value is fixed when changing the scale, rotation, blurring, lighting, and also changes shape. This shape change occurs because the shape of the query image that is not intact or not as perfect as the image that is in the image database. Query incomplete picture possible because another object covering, or shooting is not perfect, or the state of the object image itself that has changed. SURF algorithm produces high-dimensional image features that can be compared with other image features. Features of SURF can be used to indicate if there is a relationship resemblance to an image area in a different image.

\section{Research Method}

SIFT and SURF algorithm is applied to stitching aerial photography, process steps are carried out almost the same. The process that is different from the system is when its extraction process. As for the search process as well as the suitability of incorporation using the same method.

The system design stitching aerial photographs using SIFT and SURF algorithm can be seen in Figure 1. Diagram block system. This aerial photograph stitch program is designed to be able to process the picture obtained from the UAV fixed-wings with a limited image display, to an output device that produces a broader view by combining with another image that corresponds.

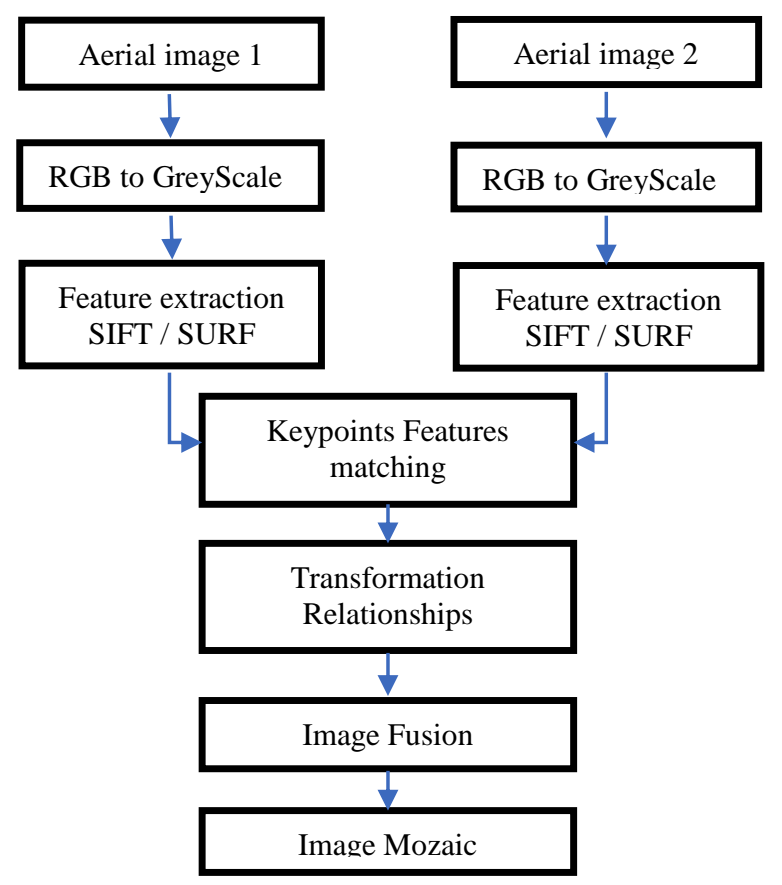

Figure 1. Diagram Block System 


\section{Result and Analysis}

There are several stages to test stitching a system of images aerial using the algorithm SIFT and SURF, among other things, the minimum testing point of features that can be stitched, testing of the minimum percentage of the intersection of images that can be stitched, testing image rotation and image scale testing. Figure 2 illustrates the test of image variation.
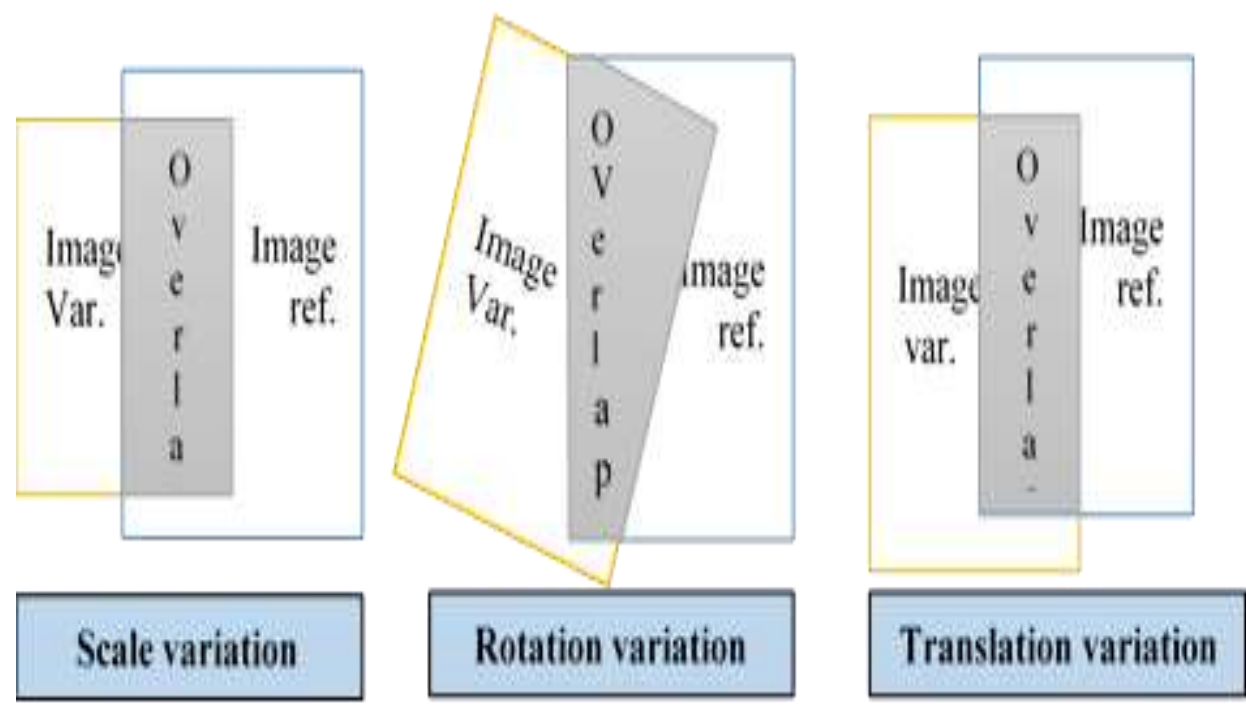

Figure 2. Illustration Testing of Image Variations

\subsection{Points of Features Testing}

Testing is done by cutting the image into two parts to form an area of overlap between the images and then made to match the image of the second points of features. Testing varied in order to obtain the minimum points of features so that the second image can still be stitched. The results of testing with methods SURF and SIFT points of features obtained a minimum of 5 .

\subsection{Overlap Minimal Testing}

Testing is done by cutting an image into two sections with a percentage variation of the different intersection. Images stitching can be seen in Table 1.

Table 1. Testing minimum overlap area using SURF is the result of the test on the system using the aerial image merging algorithms SURF to test how minimal the point of intersection of the two inputs that can still be incorporated using a system that has been created. The system is able to perform the image stitching to the percentage of overlap between the images by $50 \%, 30 \%$. As for the overlap of $10 \%$ is not able to produce a perfect image because of the lack of intersection of the two image inputs that cause system points of features found little in common between the two inputs. While the results of testing, the system using SIFT algorithm, the system is still able to perform stitching areas overlapping images although only $4 \%$. 
Table 1. Testing Minimum Overlap Area using SURF

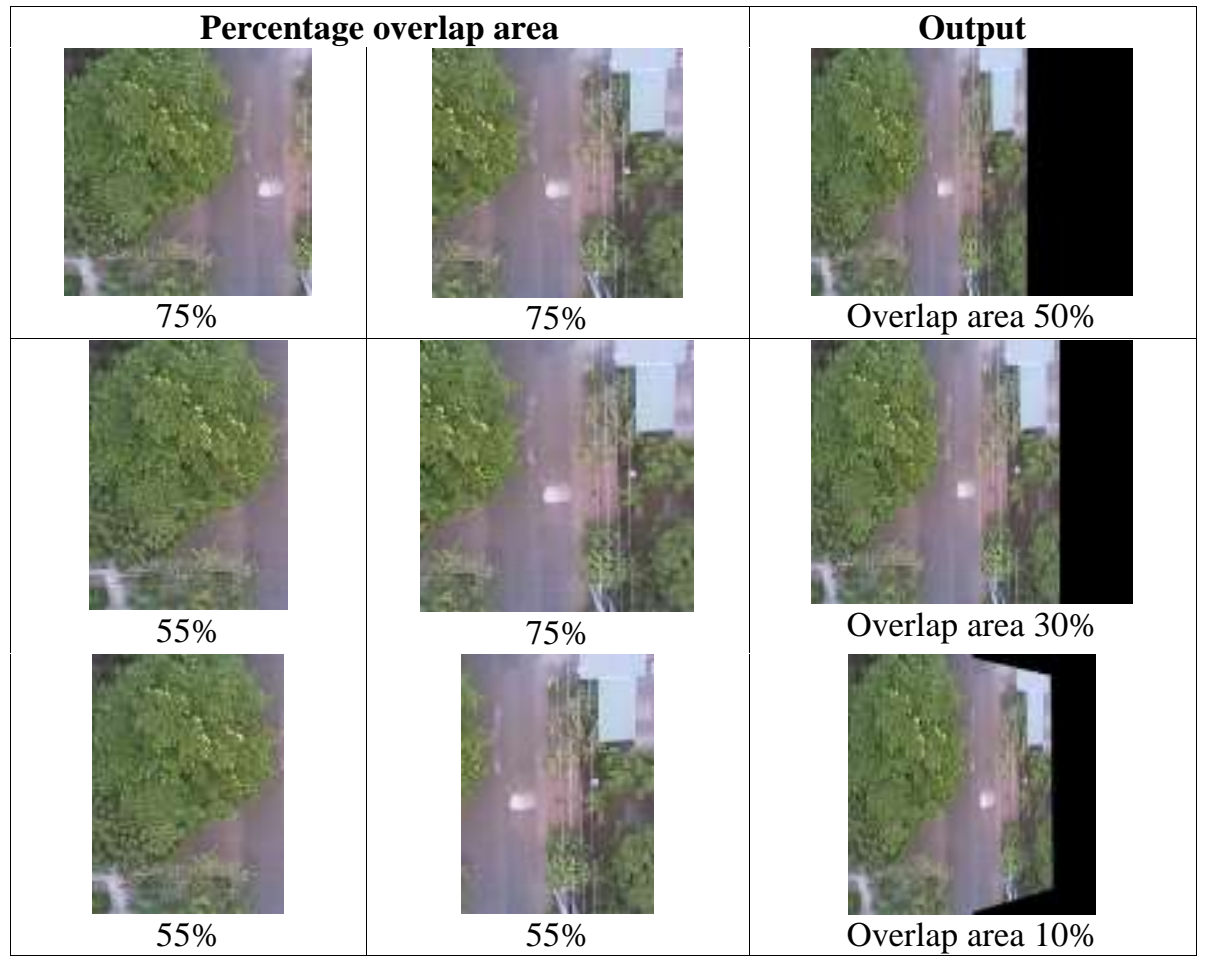

\subsection{Rotation Testing}

Testing is done by rotating one of the input images with some variation of the angle of rotation. The angle of rotation is done variation is $20^{\circ}, 90^{\circ}, 180^{\circ}$, and $270^{\circ}$.

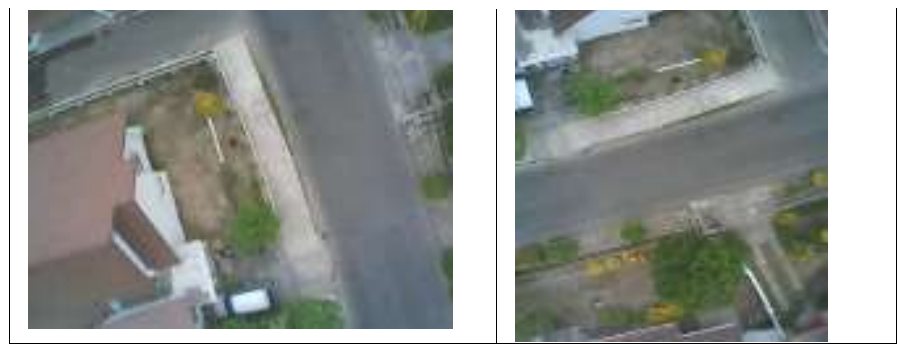

Figure 3. Image 1 (left) Image 2, Rotate $90^{\circ}$ (right)

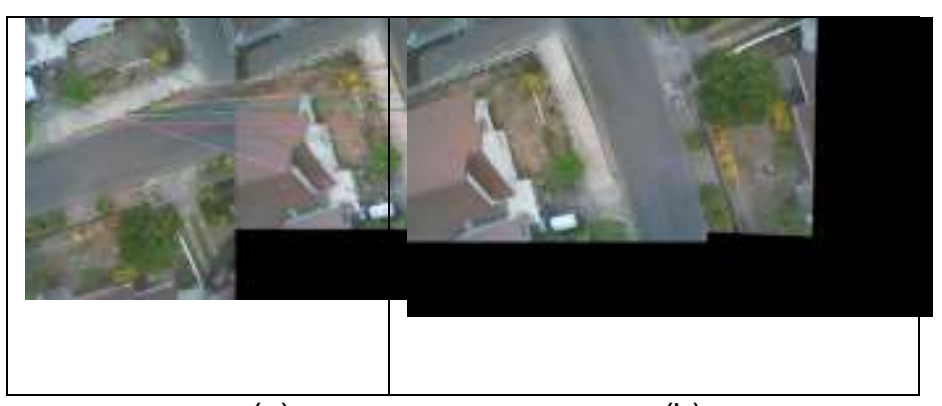

(a)

(b)

Figure 4. (a) Key Points Matching Figure 3(b) Stitching Image

Figure 3. is a second input to the image with one of the inputs made by way of variation of the angle of $90^{\circ}$ rotates clockwise. For the first input is made normal, 
resulting in points of features matching results as in Figure 4. (a) SURF algorithm still detects points of features despite experiencing rotational position of the image, so it can be searched the same points through points of features similarities between the two input image. Figure 4. (b) shows the output image that has been incorporated, using homographic matrix calculations performed by RANSAC algorithm. Then merging technique using warp Perspective, this technique to transform the perspective in an image that is similar to the first image and then performed for the image buildup same points of features between the two inputs. Therefore, the image can be combined though with different input angle rotated because SURF can detect any points of features although changing rotation. Warp Perspective method can also perform perspective transformation so that the viewpoint of the second input is made similar to the first input viewpoint. While testing using SIFT method, the system is able $\left(0^{\circ}-360^{\circ}\right)$ to stitching the image with various positions of rotation between images.

\subsection{Testing of Scaling}

Testing is done by changing the scale or the size of one input to a size that is different from the first input. The first test performed on a scale of variation of 50\% smaller than its actual size.

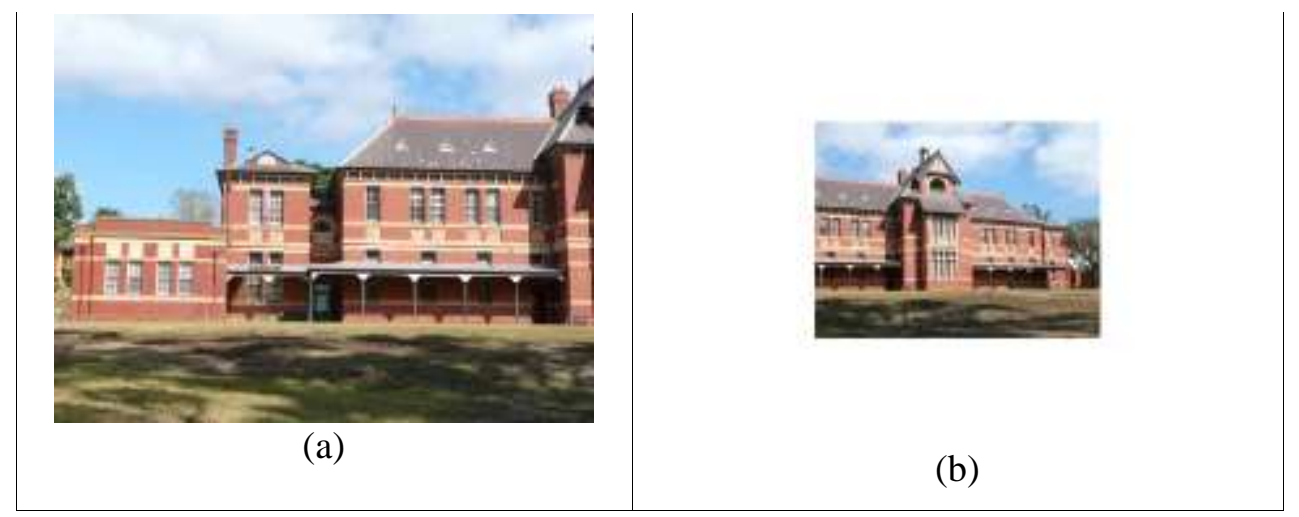

Figure 5. Scale 50\%

Figure 5. is a test of the scale which has a scale ratio of $50 \%$, the image (a) has a normal size, then the image (b) have a size smaller scale 50\% of the actual size. In this test still found keypoint matching between the two inputs, although the amount is not as much the same keypoint inputs that have the same scale ratio. Figure 6 . shows the output for matching keypoint between the two inputs with a variation scale 50\%. Then Figure 7. shows the results of merging images for differences in scale, the results can be seen that the image produced is a bit blur to images scaled back, because in the process of merging performed a small image magnification adjusted to a large scale at the first input. 


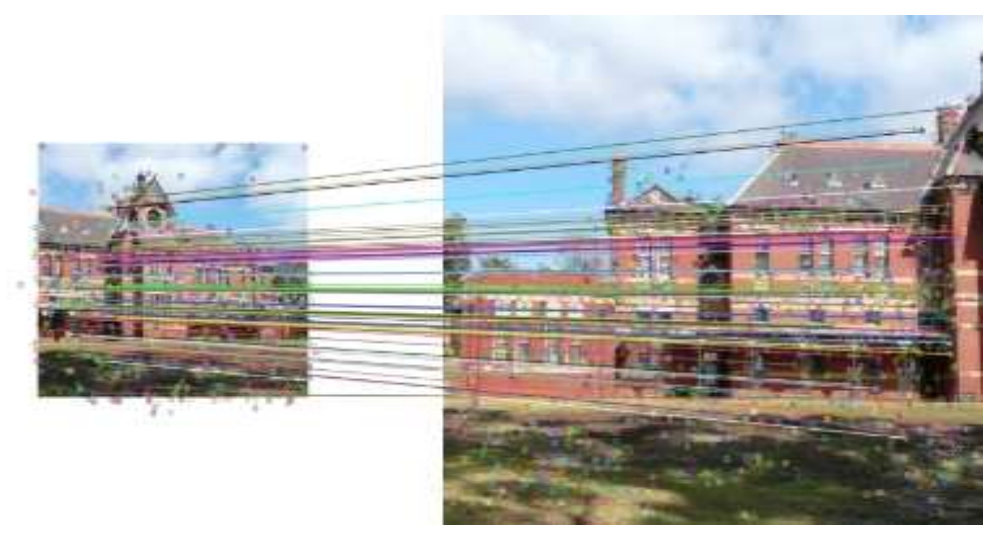

Figure 6. Keypoint Matching Results in Scale 50\%

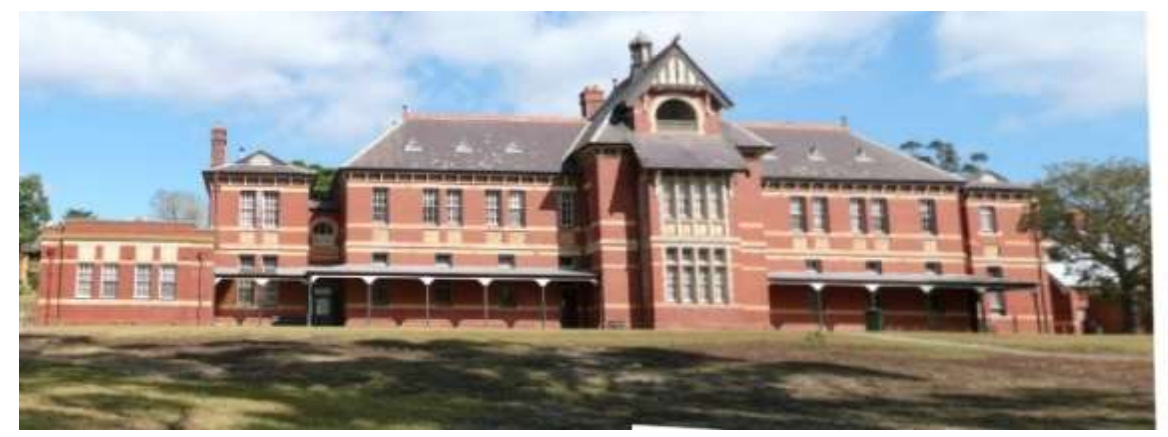

Figure 7. Result Stitching For Scale 50\%

Testing of the scale of variation values obtained for the smallest scale can be combined is $25 \%$ of the original image. If the image is reduced more than the value of the process cannot be merged. For test scale of an enlarged, done by enlarging the scale on one of the inputs. Figure 8. Image input for Scale $200 \%$ shows the variation of one of the inputs with inputs enlarged to $200 \%$.

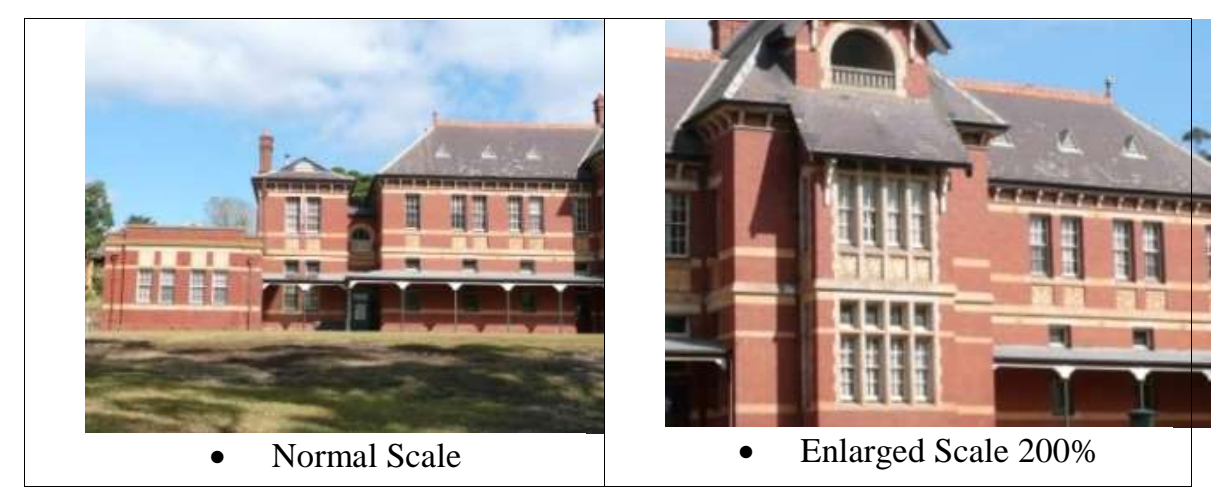

Figure 8. Image Input for Scale 200\% 


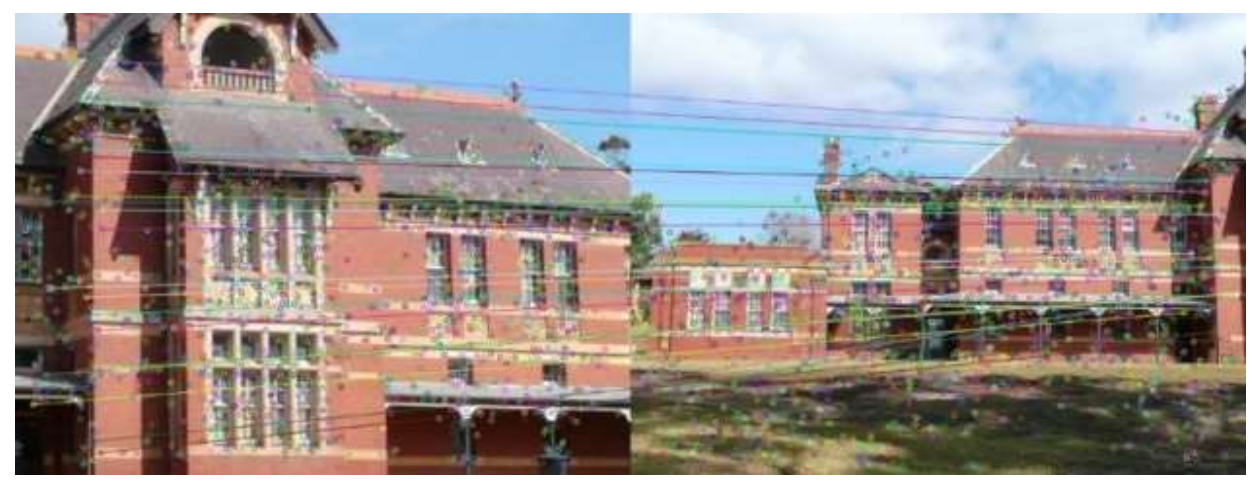

Figure 9. Keypoint Matching for Scale 200\%

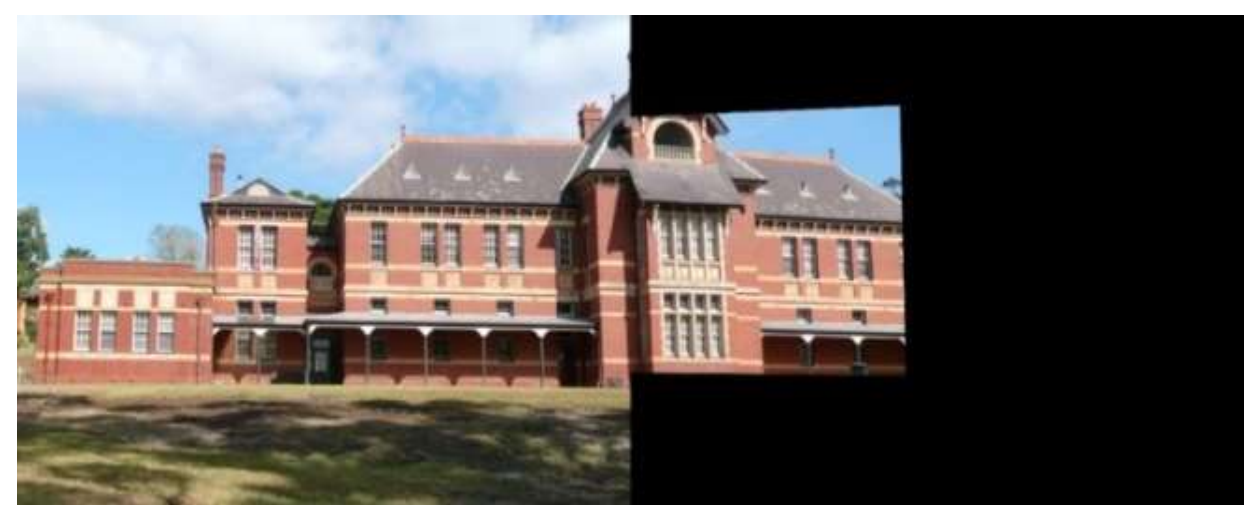

Figure 10. The Result Stitching for Scale 200\%

Testing of on the magnification of $200 \%$ may still be a possible stitch. Figure 9 . Keypoint matching for scale $200 \%$ shows that although the image scale is different between the two inputs, still can be done matching keypoints which accords due SURF features can still find keypoints despite a change in scale. Figure 10. The result stitching for scale $200 \%$ shows the result of merging two images with different scale values. On the left picture looks like a truncated, it happened because of the magnified area of the original image to produce a smaller viewing area, then the image is enlarged experiencing downsizing scale adjusted to the scale of the first image input.

Testing of variations scale get the most scale magnification images that can be stitched is $600 \%$ enlarged from the original image. More than that the image has not be stitched because it will produce a number keypoints less than 5 , the amount that can be stitched more than 5 keypoints. Magnification scale $650 \%$ will produce 4 keypoints so that the image cannot be stitched. The result of the comparison can show in Table 2.

Table 2. Comparison of SIFT and SURF For Stitching Image Aerial

\begin{tabular}{|l|c|c|}
\hline Parameter Testing & SIFT Algorithm & SURF Algorithm \\
\hline Keypoints & 5 & 5 \\
\hline $\begin{array}{l}\text { Minimum overlap } \\
\text { area }\end{array}$ & $4 \%$ & $15 \%$ \\
\hline Rotation & $0^{\circ} \sim 360^{\circ}$ & $0^{\circ} \sim 360^{\circ}$ \\
\hline Scale & $45 \% \sim 480 \%$ & $25 \% \sim 600 \%$ \\
\hline
\end{tabular}




\section{Conclusion}

This paper presented Comparative of SIFT and SURF for stitching image aerial. Both methods can be used for the incorporation of images aerial. Both methods are resistant to changes in the rotation and scaling, and simply use areas overlap slightly so that the difference in altitude when taking images aerial still allows images aerial stitched. The results comparison of the two methods shows that the difference in height and the area of overlap slightly (4\%) and scale difference between images by $45 \%$ to $480 \%$ by SIFT and $25 \%$ to $600 \%$ by SURF are still possible to process image aerial stitching.

\section{References}

[1] D. G. Lowe, "Object Recognition from Local Scale-Invariant Features", Proceedings of the Seventh IEEE International Conference on Computer Vision, (1999), pp. 1150-1157.

[2] D, G. Lowe, "Distinctive image features from scale-invariant keypoints", International Journal of Computer Vision, vol. 60, no 2, (2004), pp. 91-110.

[3] D.G. Lowe, "Local feature view clustering for 3D object recognition", IEEE Conference on Computer Vision and Pattern Recognition, (2001), pp. 682-688.

[4] Z. Yinhai, "A Novel SVPWM Modulation Scheme", in Applied Power Electronics Conference and Exposition, 2009. APEC 2009. Twenty-Fourth Annual IEEE, (2009), pp. 128-131.

[5] Q. T. Luong and O. D. Faugeras, "The fundamental matrix: Theory, algorithms, and stability analysis", International Journal of Computer Vision, vol. 17, no. 1, (1996), pp. 43-76.

[6] J. Beis and D. G. Lowe, "Shape indexing using approximate", Conference on Computer Vision and Pattern Recognition, (1997)1, pp. 000-1006.

[7] K. Mikolajczyk and C. Schmid, "An affine invariant interest point detector", European Conference on Computer Vision, (2002), pp. 128-142.

[8] H. Bay, T. Tuytelaars and L. Van Gool, "SURF: Speed Up Robust Features", pp. 1-14.

[9] L. Juan and O.Gwun, "SURF Applied In Panorama Image Stitching", Image Processing Theory, Tools And Applications, IEEE, (2010).

[10] Y. Liang, Q. Li, Z. Lin and D. Chen, "A Panoramic Image Registration Algorithm Based On SURF", Springer-Verlag Berlin Heidelberg 2012, recent advances in CSIE, LNEE, vol. 128, pp. 473-478.

[11] H. Bay, A. Ess, T. Tuytelaars, L. Van Gool, "Speed Up Robust Features (SURF)", Elsevier, (2008). 